\title{
New Approach to Integration of Form Deviations in the Analysis of Geometric Tolerances
}

\author{
Mouhssine Chahbouni ${ }^{* 1}$, Mustapha Elmouden ${ }^{* 2}$, Said Boutahari ${ }^{* 3}$, Driss Amegouz ${ }^{* 4}$ \\ *Laboratory of Manufacturing, Energy and Sustainable Development (LPEDD) \\ High School of Technology, BP 2427 Road of Imouzzer 30000 Fez, Morocco \\ ${ }^{1}$ mouhssine.chahbouni@usmba.ac.ma \\ ${ }^{2}$ mm.mouden@gmail.com \\ 3boutahari@yahoo.fr \\ 4amegouz@yahoo.fr
}

\begin{abstract}
Tolerancing is an important step in the design process of a mechanism. There are two tolerancing approaches: tolerance analysis and tolerance synthesis. The principle of tolerance analysis is to verify the functional condition of the mechanism, for that purpose it is necessary to check the specific tolerances of each elementary part. Whereas the tolerance synthesis aim to calculate the specific tolerances of the parts forming the mechanism. The purpose of this paper is to develop a new approach of geometric tolerances analysis with integration of form deviations. For this we studied an example of assembly of two cylindrical parts (shaft and bore). The first step is to analyze the tolerances of the component parts assembly using the deviation domain method by neglecting the form deviations. The second step of this study is to analyze the tolerances with the same method but with the introduction of the form deviations, by using the discrete modal method for the modeling of surfaces with form deviations and applying a new algorithm developed for the simulation of our approach. Finally, we compared the two studies with and without form deviations through the calculation of a non-compliance rate (TNC)
\end{abstract}

Keywords - Geometric Tolerancing, Tolerance Analysis, Domain Method, Discrete Modal Method;

\section{INTRODUCTION}

Tolerance analysis is a process of estimating the propagation of specific tolerances of elementary parts in a mechanism. The objective is to optimize the product's manufacturing cost while ensuring functional condition.

We study in this paper an example of assembly of a shaft with its bore. First, we present an analysis of the geometric tolerances with the method of the domains without integration of form deviations. In a second step we present a new approach of tolerance analysis with integration of form deviations, by using the method of discrete modal decomposition for the form deviations modeling, and then we apply a new algorithm for the simulation of the analysis with integration of form deviations. Finally, we compare the results with and without form deviations.

\section{Domains Method}

The domain method allows the geometric tolerances analysis of mechanical systems. The dimensional and geometric tolerances of the parts being defined, this method allows to define the relations between functional tolerances and specific tolerances to each linking or functional surface. This method has been developed and applied to mechanisms with serial and parallel links [1] [2].

This approach is based on the theory of small displacements torsor, allows to specify the deviations domains and the gaps domains [3] [4].

\section{DeViation Domain}

Any surface has 6 deviations components: 3 translations and 3 rotations. These deviations are small amplitudes relative to the relative positions of the surfaces, it is possible to characterize them by a small displacements torsor where $\delta \theta$ is the rotation vector and $\delta(\mathrm{M})$ is the translation vector[5] ,[6].

$$
\{\overrightarrow{\delta(\mathrm{M})}\}_{M}=\left(\begin{array}{cc}
\mathrm{r}_{\mathrm{x}} & \mathrm{t}_{\mathrm{x}} \\
\mathrm{r}_{\mathrm{y}} & \mathrm{t}_{\mathrm{y}} \\
\mathrm{r}_{\mathrm{z}} & \mathrm{t}_{\mathrm{z}}
\end{array}\right)
$$




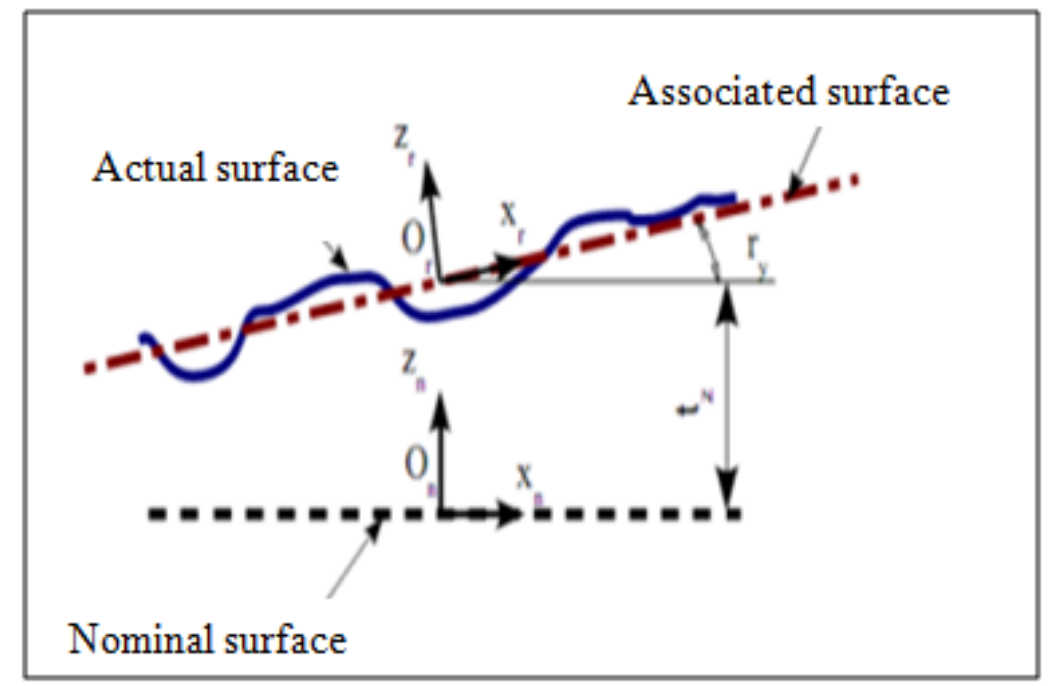

Fig. 1. A flat surface deviations

\section{IV.DisCRETE MODAL DECOMPOSITION METHOD (DMD)}

The DMD is based on the vibration theory of discretized mechanical structures. Each natural mode of vibration defines a particular geometry. These modes are used as parameters defining the form deviations of a surface [7]. These modes of vibration are calculated from equation 2 :

$$
\text { M. } \frac{\partial^{2} \mathrm{u}}{\partial \mathrm{t}^{2}}+\mathrm{K} \cdot \mathrm{u}=0
$$

Where $\mathrm{K}$ and $\mathrm{M}$ are matrices of rigidity and mass, and $\mathrm{u}$ is the displacement vector.

The solution of equation (2) provides a linear system whose solutions are the natural mode of vibration Qi corresponding to the pulsation $\omega$ i.

$$
\left(M^{-1} \cdot K-\frac{1}{\omega_{\mathrm{i}}^{2}} \cdot I\right) \cdot Q_{i}=0
$$

Each natural vector is normalized according to the infinite norm so that $\left\|\mathrm{Q}_{\mathrm{i}}\right\|_{\infty}=1$.

To generate surfaces with form deviations, it is necessary to discretize the surface in finite elements and to compute in a first time the natural modes of this surface, then generate the modal coefficients. Each surface is calculated with the following equation:

$$
\mathrm{S}=\mathrm{Q} . \mathrm{m}
$$

Where $\mathrm{Q}$ is the matrix of the natural modes of the surface and $\mathrm{m}$ is the modal coefficients vector.

\section{TOlerancing OF AN ASSEMbly (ASSEMbly OF A SHAFT With ITS Bore)}

The example studied is an assembly of two cylindrical parts. The functional condition to verify is the assemblability of the shaft with the bore, which is represented by a geometric tolerance of coaxiality. The assembly and definitions drawing of parts are illustrated in figure 2.

Hypotheses

- The parts are rigid.

- The study of parts concerns only the functional surfaces 


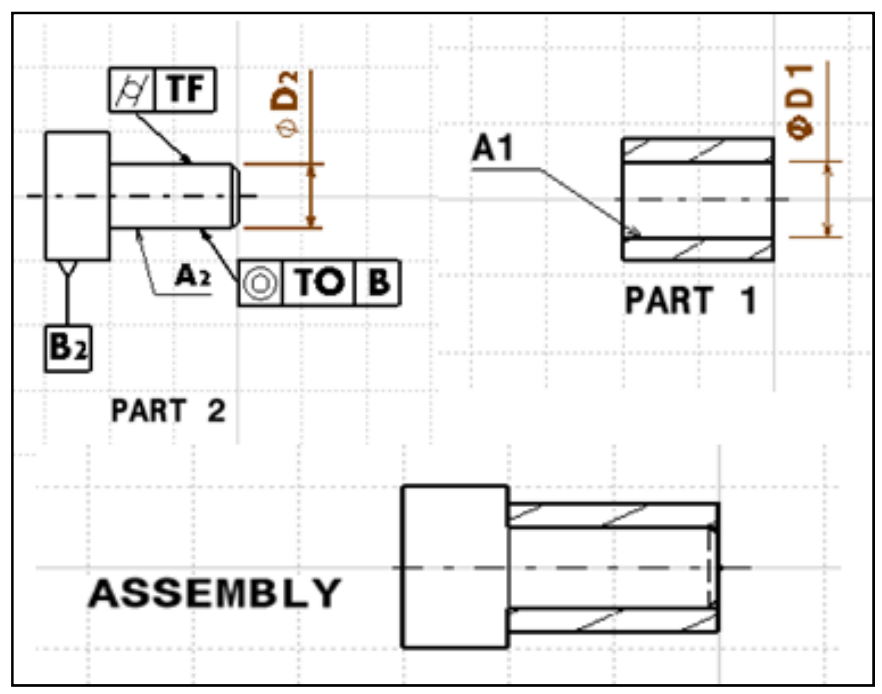

Fig. 2. Example drawing

\section{A. Tolerancing without Introduction of Form Deviations.}

Study of deviation domains.

- Study of the part (1): Bore

The part (1) is considered as a reference part for the assembly, so the deviation domain of the cylindrical surface (A1) is zero: $\mathrm{E} 1 \mathrm{~A}=0$.

- Study of the part (2): Shaft

The surface (B2) of the part (2) is a reference surface of coaxiality tolerance of the surface (A2), so the deviation domain of this surface is zero: $\mathrm{E} 2 \mathrm{~B}=0$

The surface (A2) is a cylindrical surface toleranced in coaxially with related to the surface (B2), so the deviation torsor of this surface is of the following form:

$$
\mathrm{E} 2 \mathrm{~A}=\left\{\begin{array}{cc}
\mathrm{r}_{\mathrm{x}} & \mathrm{t}_{\mathrm{x}} \\
\mathrm{r}_{\mathrm{y}} & \mathrm{t}_{\mathrm{y}} \\
0 & 0
\end{array}\right\}_{\left(0, \mathrm{x}_{0}, \mathrm{y}_{0}, \mathrm{z}_{\mathrm{o}}\right)}
$$

To respect the coaxial tolerance assigned to the cylindrical surface (A2), the displacement of the points $\mathrm{O} 1$ and $\mathrm{O} 2$ along the two axes $\mathrm{X}$ and $\mathrm{Y}$ must not exceed the tolerance interval divided by 2 (to / 2 ).

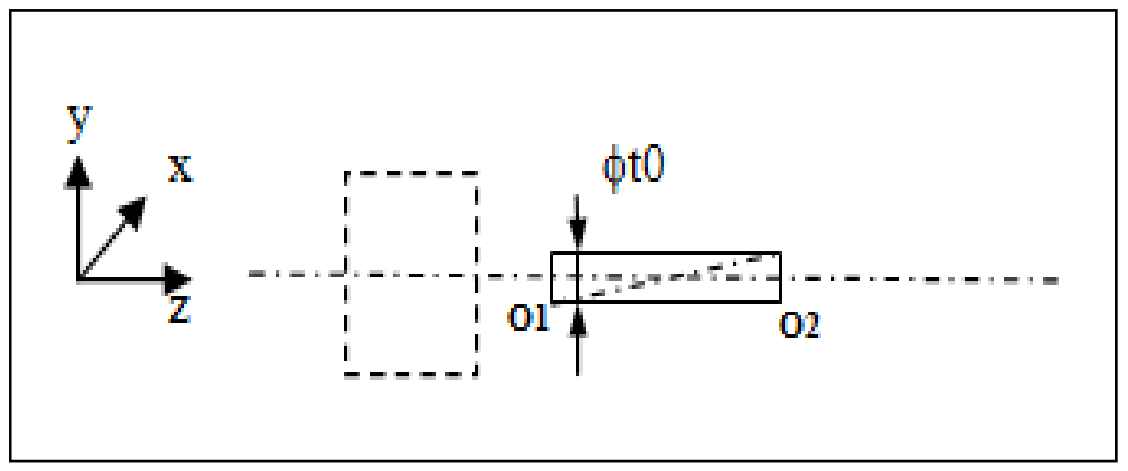

Fig.3. Presentation of the tolerance zone of coaxiality

$$
\left\{\begin{array}{l}
-\frac{\text { to }}{2} \leq \overrightarrow{\delta 01} \cdot \vec{x} \leq \frac{\text { to }}{2} \\
-\frac{\text { to }}{2} \leq \overrightarrow{\delta 01} \cdot \vec{y} \leq \frac{\text { to }}{2} \\
-\frac{\text { to }}{2} \leq \overrightarrow{\delta 02} \cdot \vec{x} \leq \frac{\text { to }}{2} \\
-\frac{\text { to }}{2} \leq \overrightarrow{\delta 02} \cdot \vec{y} \leq \frac{\text { to }}{2}
\end{array}\right\}
$$

The deviation domain of this surface in the center of the cylindrical link is of the following form: 


$$
(E 2 A) \text { au point } O=\left\{\begin{array}{l}
-\frac{t o}{2} \leq t_{x}+\frac{h}{2} r_{y} \leq \frac{t o}{2} \\
-\frac{t o}{2} \leq t_{x}-\frac{h}{2} r_{y} \leq \frac{t o}{2}
\end{array}\right\}
$$

The graphical representation is of the following form:

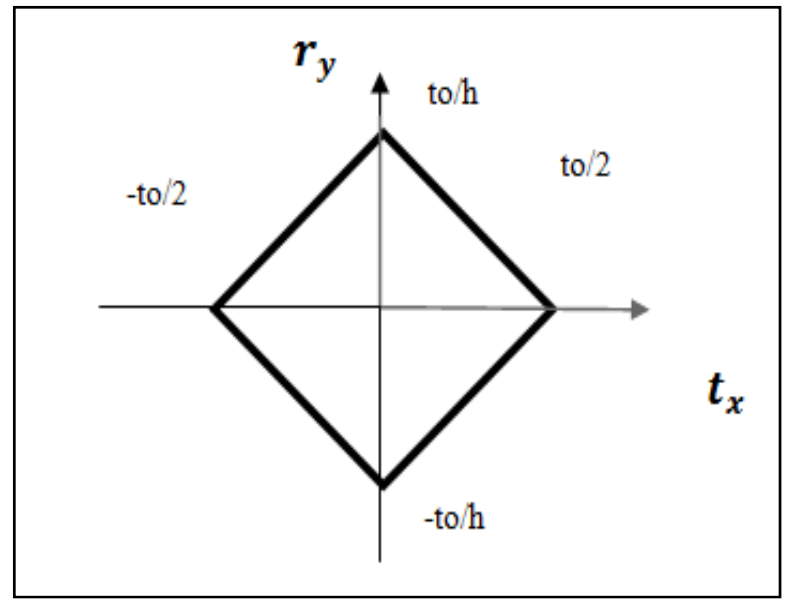

Fig. 4. Representation of the deviation domain of coaxiality E2A

- Study of the functional condition of the assembly

It is considered that the tolerance of coaxiality represents the functional condition of the assembly, so for the assembly to be functional it suffices to respect this tolerance during the manufacture of the parts.

\section{B. Introduction of Form Deviations}

Several studies have studied the integration of form deviations in assemblies, especially in the case of flat surfaces [8]. In our case, we studied an assembly of two cylindrical parts. [9], [10], [11], [12], [13] [14], [15], [16].

Calculation of natural modes of the studied surface

The two surfaces in contact in our assembly are cylindrical surfaces. The natural modes of this surface are calculated. To simplify the study, we consider that the cylindrical surface is represented with its axis with the deformations caused by vibrations. The following figure shows some natural modes of a cylindrical surface.

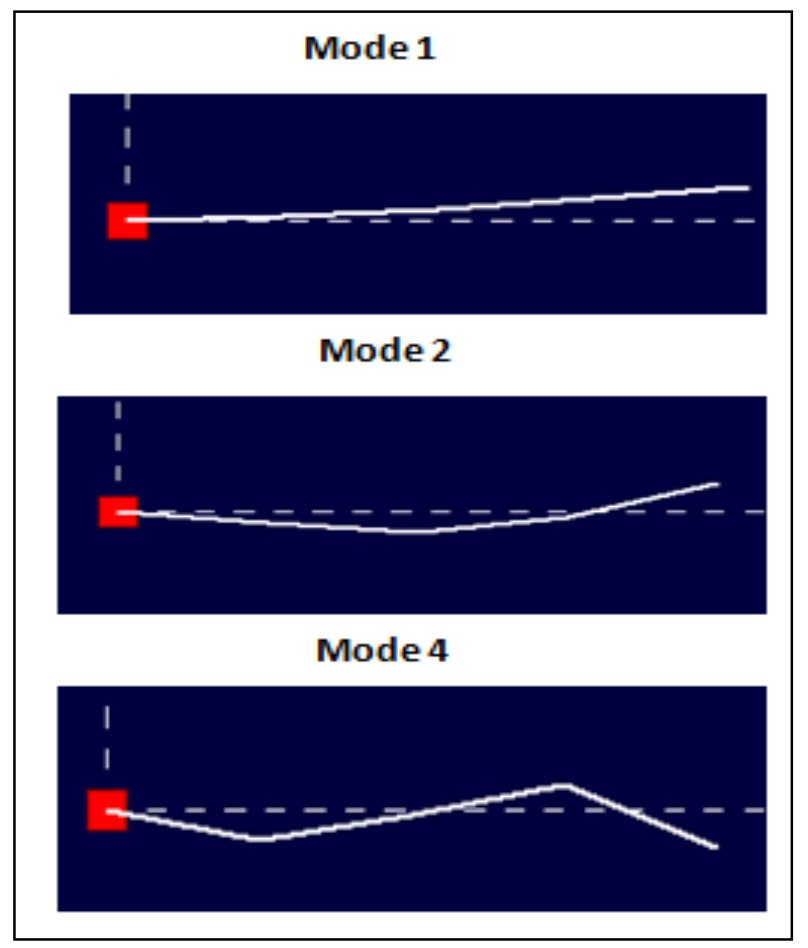

Fig.5. Natural mode of the cylindrical surface 
Generations of surfaces with form deviations

Each surface is modeled with a set of nodes that undergo displacements along the y axis, the displacements of all the nodes of all the natural modes are normalized so that the displacements are between "-1" and "1". Then we generated for each natural mode 20 random values according to the normal law between 0 and 1 . In our case each surface is composed of 5 nodes. The calculation is done using the following equation using "Excel ":

- S1.1 : Displacement of node 1 of surface 1

$$
\left[\begin{array}{c}
\mathrm{S} 1.1 \\
\mathrm{~S} 1.2 \\
\vdots \\
\mathrm{S} 1.5
\end{array}\right]=\left[\begin{array}{ccc}
\mathrm{Q} 1.1 & \cdots & \mathrm{Q} 3.1 \\
\vdots & \ddots & \vdots \\
\mathrm{Q} 1.5 & \cdots & \mathrm{Q} 3.5
\end{array}\right] \cdot\left[\begin{array}{c}
\mathrm{m} 1.1 \\
\mathrm{~m} 1.2 \\
\vdots \\
\mathrm{m} 1.3
\end{array}\right]
$$

- Q1.1 : Value of mode 1 for node 1 of surface $\mathrm{i}$

- m1.1 : Random coefficient for mode 1 of surface 1

We generated 20 random surfaces form deviations based on the natural modes of vibration of the cylindrical surface. The following figure shows the calculated surfaces with form deviations.

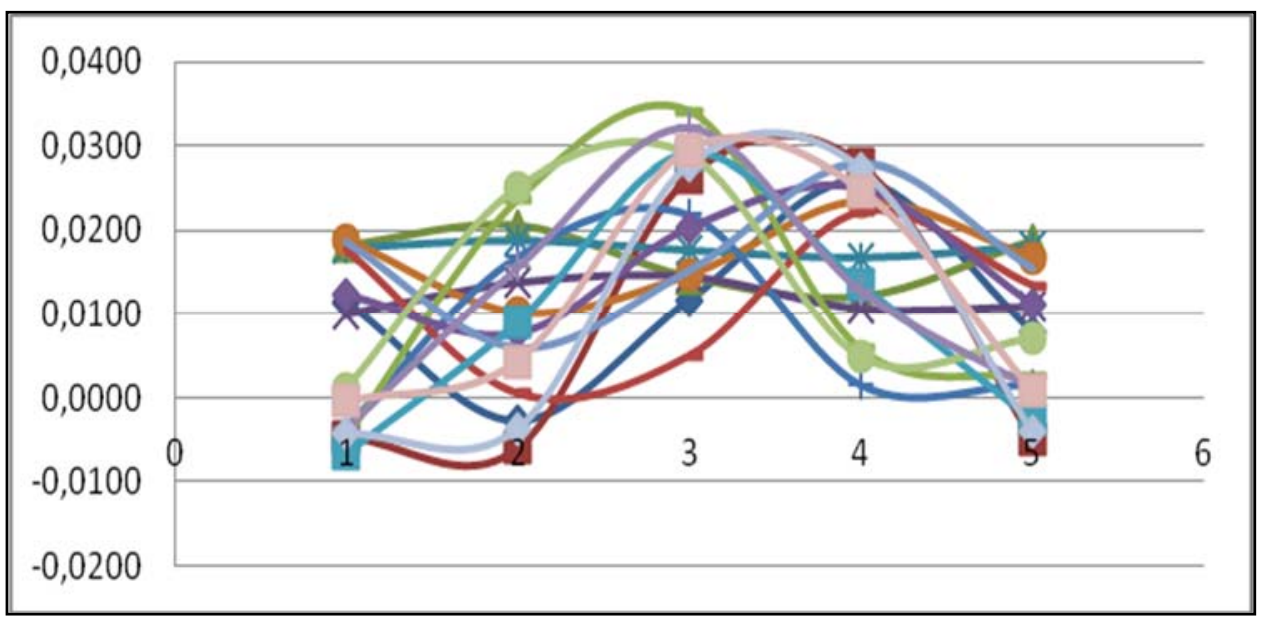

Fig.6. Representation of surfaces with form deviations

Finally, we calculated the form deviations of the generated parts and we selected 16 values that are within the tolerance interval of the form deviations imposed by the designer (less than $0.04 \mathrm{~mm}$ ), then are saved in a column vector under "Excel".

Monte Carlo Simulation

- Simulation data

- Beam diameter $10 \mathrm{~mm}$, length $\mathrm{a}=10 \mathrm{~mm}$

- Materials: Young's module stainless steel $\mathrm{E}=203000 \mathrm{Mpa}$

- 5 nodes with embedding link on node number 1.

- Coaxiality tolerance : to $=0.2 \mathrm{~mm}$

- Dimensions : $\mathrm{a}=\mathrm{b}=10 \mathrm{~mm}$

- $\quad$ tx: according to the normal distribution of mean $(\mathrm{m}=0)$ and standard deviation $(\delta=33.66 \mu \mathrm{m})$

- ry: according to the normal distribution of mean $(\mathrm{m}=0)$ and standard deviation $(\delta=3.33 .10-3 \mathrm{rad})$

- $\quad \mathrm{tf}$ : this is the form deviation tolerance, which is randomly chosen from the vector of generated form deviations values

- $\mathrm{r}=0.2$ : it is the ratio between the form deviations and the orientation deviations

- $\quad \mathrm{c} 1$ : counter of accepted parts taking into account form deviations

- c2: counter of accepted not accepted parts with form deviations

- $\quad \mathrm{c}=\mathrm{c} 2 /(\mathrm{c} 1 \mathrm{c} 2)$ : this is the rate of non-conformity

- Simulation steps

- Generation of the random variables rx, ry and $\mathrm{tf}$ : to each part $\mathrm{i}$ we associate three random variables rxi, ryi and tfi according to a normal distribution.

- Calculation of the control parameters of the part: for each part i, the displacements of the extreme points of the toleranced surface are calculated. 
- Verification of the conformity of the part taking into account the form deviations: for the part to be accepted, the control parameters must stay within the tolerance interval.

- Representation of the conforming parts with the consideration of form deviations: each piece i conforms is represented with a blue point of coordinates (txi, ryi).

- Verification of the conformity of the parts without taking into account the form deviations and which are rejected with the taking into account form deviations: in order for the part to be conforming, it is necessary that the parameters of control stay within the tolerance interval without form deviations.

- Graphic representation of these parts: each part $\mathrm{i}$ is represented with a red star of coordinates (txi, ryi).

- Calculation of the non-conformity rate: this is the ratio between the number of parts conforms with the consideration of the orientation deviations and the form deviations divide by the number of parts conforms without taking into account form deviations.

- Execution of the algorithm and interpretation of the result

After executing the program of this simulation, we obtain the result illustrated in the following figure:

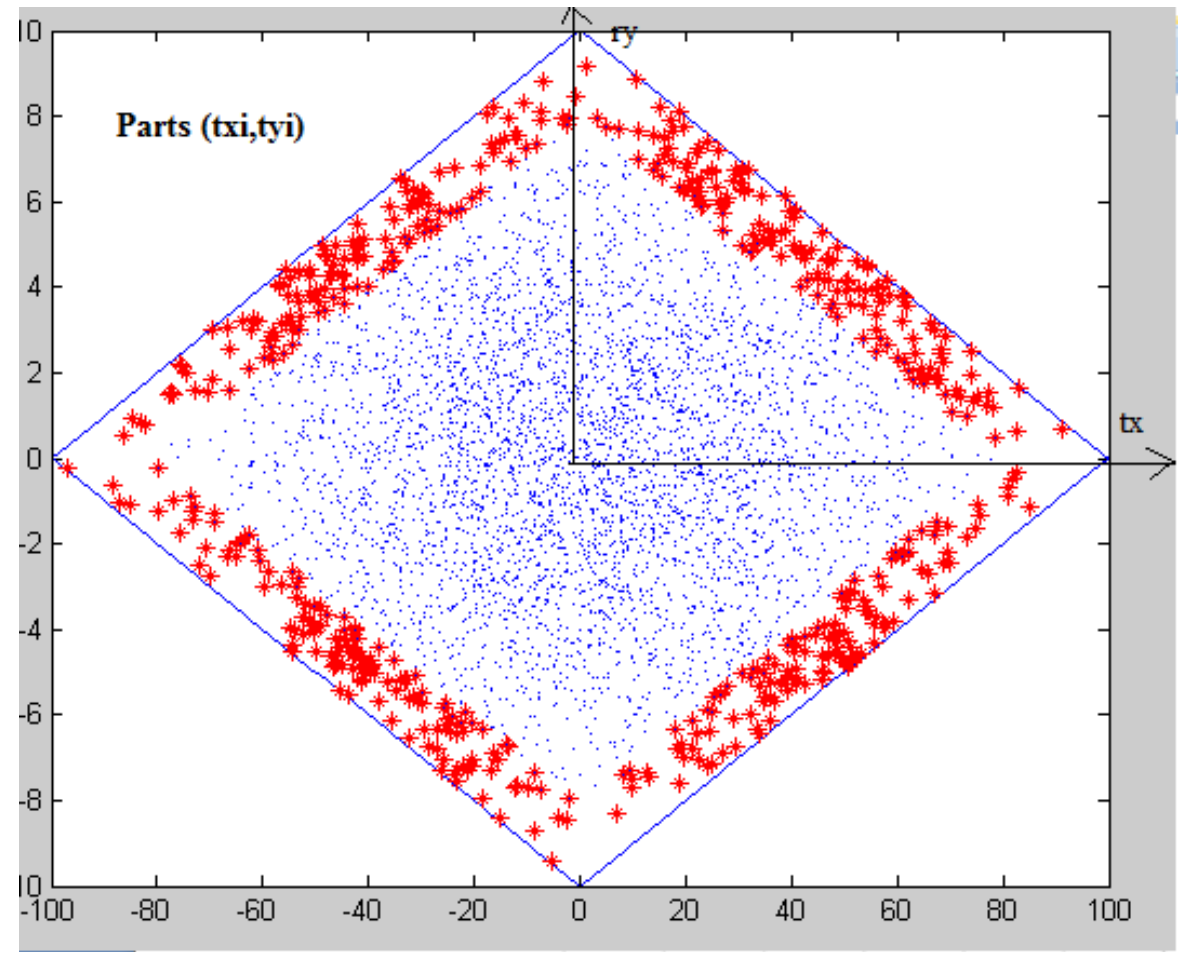

Fig.7. Result of the simulation with form deviations

The parts represented with blue points are accepted with the taking into account of the two types of deviations: the coaxiality and the form deviations. The parts represented with red stars are defective parts if we take into account the form deviations, whereas they are accepted if we neglect the form deviations. To illustrate the influence of the introduction of the form deviations on the tolerance analysis with the domain method, we calculated the rate of non-conformity which is of the order of $5 \%$.

\section{VI.CONCLUSION}

In this paper we studied through an example of assembly of two cylindrical parts, the geometric tolerances analysis with and without taking into account the form deviations. The first part presents the tolerancing without taking into account form deviations, by using the deviations domains method with the worst-case approach. The second part presents tolerancing with consideration of form deviations. In order to model these form deviations we used the discrete modal decomposition method. Then we developed a Monte-Carlo statistical simulation for the tolerance analysis based on the new developed algorithm. Finally, the result is represented with a graph to facilitate interpretation, and then we calculated a non-conformity rate which illustrates the difference between tolerancing with and without taking into account form deviations. 


\section{REFERENCES}

[1] Petit J-P,"Geometric Product Specification: method of analysis of tolerances. Application computer-aided design" , PhD Thesis of Mechanical Engineering, University of Savoie, 2004

[2] M. Chahbouni, S. Boutahari and D. Amegouz,"Analysis of geometric tolerances by The Deviations and Clearance Domains Method", 11 th Conference of Mechanics, Agadir 2013, Morocco.

[3] P.BOURDET and A.CLEMENT,"controling a complex surface with a 3 axis measuring machine", annals of the CIRP, Vol 25, Manufacturing technology P 359, Janvier 1976.

[4] P.BOURDET , L. MATHIEU ,C. LARTIGUE and A. BALLU, "The concept of the small displacement torsor in metrology", proceeding of international euro conference advanced mathematical tools in metrology ,oxford 27-30 September 1995

[5] E.PAIREL,"Proposition of a new semantic for standardized tolerancing _ principle of its verification on coordinate measuring machines" , 7th International Congress of Metrology, PP 76-81, 1995.

[6] Frederic GERMAIN, "Three-dimensional statistical tolerancing, integration in CFAO”, PhD University of Savoie, October 2007.

[7] Samper, S. and Formosa, F. Form defects tolerancing by natural modes analysis. J. Computer. Inf. Sci. Eng., 2007, 7(1), 44-51.

[8] Stephane Morière, "contribution to the study of joints with consideration of the form defects: modelling and experiments", PhD of the University of Mediterane 2010

[9] J. Grandjean, Y. Ledoux and S. Samper,'Influence the form defects in joints flat surfaces", the 20th Congress of Mechanics French, Besançon, 2011.

[10] Gupta ,S.ans Tourner, J.U , variational solid modling for tolerance analyses , IEEE computer, graphic application 1993 , $13(3)$, 64-74.

[11] Huang, W. and Ceglarek, Mode-based decomposition of part form error by discrete-cosine-transform with implementation to assembly and stamping system with compliant parts. CIRP Ann., 2002, 51, 21-26.

[12] M.Chahbouni, Contribution to the design of mechanisms: tolerance analysis with influence of form deviations, PhD, Sidi Mohammed Ben Abdellah University February 2016, Fez, Morocco

[13] Henke, R.P., Summerhays, K.D., Baldwin, J.M., Cassou, R.M. and Brown, C.W. Methods for evaluation of systematic geometric deviations in machined parts and their relationships to process variables, Precision Engineering., 1999, 23, 273-292.

[14] M.Chahbouni et al, influence of form deviations on the tolerances analyses, international journal of engineering \& technology , vol 3 pp 343-349, 2014,

[15] M.Chahbouni et al, Statistical tolerance analysis by integrating form deviations, international journal of innovation and applied studies ,Vol. .9 No 3 Nov.2014,pp .1281-1290

[16] M.Chabouni et al, tolerances Synthesis with influence of form deviations, Conference Conception et Production Intégrée CPI 2015 Tangier, Morocco

\section{AUTHOR PROFILE}

Mouhssine Chahbouni Received his $\mathrm{PhD}$ degree in Mechanical Engineering from The University sidi mohamed ben abdelah, fez, in 2016. Dr. Chahbouni received his Engineering Degree in Industrial Engineering from National School of Applied Sciences of Fez (ENSA - Fez), Maroc, in. Currently, Dr. Chahbouni is a Research professor working as Professor of mechanic of Department of Mechanical and Production Engineering and Industrial Maintenance at the School of Technology, fez. His areas of research include Tolerancing, tolerance analysis, optimization of tolerances in rigid and flexible mechanisms.

Mustapha Elmouden, PhD student in Mechanical Engineering from The University sidi mohamed ben abdelah, of Department of Mechanical and Production Engineering and Industrial Maintenance at the School of Technology, fez,Elmouden received her engineer degree in Mechanics Engineering from The University Abdelmalek saadi, Tangier, in 2012. Her areas of research include Tolerancing, tolerance analysis, optimization of tolerances in rigid and flexible mechanisms.

Said Boutahari received his PhD degree in Mechanical Engineering from The University sidi mohamed ben abdelah, fez, in 2007. Dr. Boutahari received his Diploma Engineer from the National School of Mineral Industry (ENIM),Maroc, in. Currently, Dr. Boutahari is a Research professor (Grade qualified teacher) in the Department of Department of Mechanical and Production Engineering and Industrial Maintenance at the School of Technology, fez. His areas of research include Tolerancing, tolerance analysis, optimization of tolerances in rigid and flexible mechanisms.

Driss Amegouz, received his PhD degree in Mechanical Engineering from The University sidi mohamed ben abdelah, fez, in 2002. Dr. Amegouz received his Diploma Engineer in Production System from National School of Engineers of Metz , France, in. Currently, Dr. Amegouz is a Research professor (Grade PES) in the Department of Department of Mechanical and Production Engineering and Industrial Maintenance at the School of Technology, fez. His areas of research include Tolerancing, tolerance analysis, optimization of tolerances in rigid and flexible mechanisms. 